\title{
Asociación entre el biotipo facial y la sobremordida. Estudio piloto
}

\author{
Association between facial biotipe and overbite. Pilot study \\ Marco Antonio Sánchez-Tito ${ }^{l, a, b}$, Emerson Elecsi Yañez-Chávez ${ }^{1, c}$
}

\section{RESUMEN}

Objetivo: Determinar la asociación entre el biotipo facial y el nivel de sobremordida. Material y métodos: La muestra estuvo constituida por 152 estudiantes entre 12 y 17 años con dentición permanente. Se tomaron registros fotográficos en norma frontal con los estudiantes en posición natural de la cabeza. Sobre las fotografías impresas se determinó el ángulo de apertura facial para establecer el biotipo facial. El registro de la sobremordida vertical se realizó de forma clínica con la ayuda de un calibrador Vernier y un lápiz dermatográfico Resultados: La distribución del biotipo facial en la muestra fue de $80,3 \%$ para los dolicofaciales, seguido por los mesofaciales con $19,7 \%$, no se encontró pacientes de biotipo braquifacial. En el grupo de dolicofaciales hubo una predominancia de sobremordida normal $(55,7 \%)$, seguido por la mordida profunda $(36,9 \%)$ y mordida abierta $(7,4 \%)$. El grupo de mesofaciales presentó mayor frecuencia de sobremordida normal $(63,3 \%)$, seguido por la mordida profunda $(33,3 \%)$ y la mordida abierta $(3,3 \%)$. No se encontró asociación estadísticamente significativa (P> 0,05). Conclusiones: Debido a que no se encontraron resultados que respondan a un patrón específico, podemos concluir que los biotipos faciales no están asociados al grado de sobremordida vertical.

PALABRAS CLAVE: Cara, desarrollo maxilofacial, clasificación, sobremordida, maloclusión. (DeCS, Bireme) 


\section{SUMMARY}

Objective: To determine the association between facial biotype and the degree of overbite. Methods: The sample consisted of 152 students, aged 12-17, with their permanent dentition. Frontal photographs were taken of the students in natural head position. Face opening angle was determined based upon printed photographs in order to establish facial biotypes. Vertical overbite was measured using a Vernier caliper and a dermatograph pencil. Results: Distribution of facial biotypes in the sample was 80.3 per cent for dolichofacials, followed by a 19.7 per cent for mesofacials, with no brachyfacial types found. Among dolichofacials, a normal overbite was found to be predominant (55.7\%), followed by a deep bite (36.9\%), and an open bite (7.4\%). Among mesofacials, a normal overbite was the most frequent finding (63.3\%), followed by a deep bite (33.3\%), and an open bite (3.3\%). No statistically significant association was found ( $P>0.05$ ). Conclusions: Since results show no specific patterns, we conclude that facial biotypes are not associated with the degree of vertical overbite.

\section{KEYWORDS: Face, maxillofacial development, classification, overbite, malocclusion. (MeSH, NLM)}

\section{INTRODUCCIÓN}

El diagnóstico en ortodoncia es un análisis crítico de las condiciones iniciales que presenta el paciente, no se trata sólo de determinar la maloclusión, sino también del estudio de un conjunto de características morfológicas que direccionan la planificación de tratamientos adecuados. A lo largo de la historia, las proporciones e índices para caracterizar las estructuras anatómicas del cuerpo humano han sido un tópico muy estudiado (1,2,3). Angle (4) en 1899 señalaba que, para que el ortodoncista sea capaz de diagnosticar correctamente las maloclusiones, debería estar familiarizado también con el estudio de las características faciales del paciente; así se podría detectar si las estructuras faciales se encuentran en armonía y en concordancia con dientes bien posicionados y una oclusión balanceada.

El concepto de biotipo facial fue descrito por Ricketts (5), quien lo definió como el conjunto de caracteres morfológicos y funcionales que determinan la dirección de crecimiento y comportamiento de la cara. La literatura es clara en señalar que la determinación del biotipo facial es fundamental para poder diseñar planes de tratamientos, debido a que la aplicación de mecánicas ortodónticas pueden generar respuestas diferentes al ser aplicadas en pacientes con similares maloclusiones pero con distintos patrones de crecimiento $(6,7)$.

Las maloclusiones se caracterizan por una alteración del crecimiento y desarrollo de los maxilares, así como por alteraciones a nivel dentario, que generan modificaciones en la forma, función y estética del sistema estomatognático $(8,9)$. La etiología es multifactorial, siendo el factor hereditario y el ambiental los de mayor importancia (10).

Schwarz (11) concluye que "no existe una conexión causal entre las maloclusiones y la arquitectura esqueletal. Cada maloclusión puede estar combinada con las más diferentes variaciones naturales de la arquitectura esquelética". Sin embargo, algunos estudios han relacionado el patrón de crecimiento facial con las maloclusiones, pero a menudo se refieren a clasificaciones de maloclusiones sagitales, sin considerar otros grupos importantes de maloclusiones $(12,13)$.

El objetivo del estudio fue determinar la existencia de asociación entre el biotipo facial de los estudiantes, evaluados por medio del ángulo de apertura facial, con el establecimiento de la sobremordida.

\section{MATERIAL Y MÉTODOS}

Estudio descriptivo relacional, de diseño no experimental y de corte transversal. La muestra estuvo constituida por 152 estudiantes de la Institución Educativa Particular (I.E.P.) Alexander Von Humboldt de la ciudad de Tacna. Los criterios de inclusión fueron: estudiantes entre 12 y 17 años de edad, en dentición permanente y completa, sin antecedente de tratamiento ortodóntico previo y sin asimetría facial evidente. Se les informó acerca de los alcances de la investigación y aprobaron su participación voluntaria por medio de un consentimiento informado. 


\section{Registros fotográficos}

Las fotografías fueron tomadas en norma frontal, con el estudiante en posición natural de la cabeza. Se solicitó a los estudiantes ubicarse a $20 \mathrm{~cm}$ de una pared blanca con los pies separados para brindarles estabilidad. Se utilizó una cámara digital compacta modelo DSC-W690 adaptada a un trípode ubicado a $150 \mathrm{~cm}$ del estudiante; todas las fotografías fueron tomadas por un solo operador (EY). Las fotografías fueron impresas en papel bond A4 y se marcaron los puntos anatómicos de tejidos blandos correspondientes a Queilon y Exocanto para determinar el biotipo facial de cada uno de los estudiantes por medio de la medición del ángulo de apertura facial. Se consideró como mesofacial aquellos estudiantes en quienes el ángulo de apertura facial fue de $45^{\circ}$ $\pm 5^{\circ}$, los estudiantes con medidas menores a $40^{\circ}$ se consideraron dolicofaciales y aquellos con medidas mayores a $50^{\circ}$, braquifaciales.

\section{Registro de la sobremordida}

Con los estudiantes en oclusión, se determinó la sobremordida marcando con lápiz dermatográfico la proyección tomada desde el centro del borde incisal de los incisivos centrales superiores sobre la cara vestibular de los incisivos inferiores; posteriormente con el calibrador Vernier se midió desde la marca hasta el borde incisal de los incisivos inferiores. En los casos donde se observó una mordida abierta, el registro fue hecho por medio del calibrador Vernier apoyándolo sobre los bordes incisales de los incisivos superiores e inferiores. Los datos fueron registrados en la ficha de observación.

El análisis estadístico fue realizado por medio del programa SPSS para Windows en su versión 17. Se Empleó estadística descriptiva y estadística analítica por medio de la prueba Xi cuadrado fijando un nivel de significancia de 0,05 que corresponde a un intervalo de confianza del $95 \%$.

\section{RESULTADOS}

De los 152 estudiantes evaluados, el $60,5 \%$ ( $n=$ 92) fue de sexo masculino y el $39,5 \%(n=60)$ de sexo femenino. La prevalencia de las maloclusiones verticales fue la siguiente: $57,2 \%(\mathrm{n}=87)$ presentó sobremordida normal, $36,2 \% \quad(n=55)$ mordida profunda y $6,6 \% \quad(n=10)$ mordida abierta. Con respecto a la distribución del biotipo facial: $80,3 \%$ $(n=122)$ correspondió a estudiantes dolicofaciales y $19,7 \%(n=30)$ a mesofaciales; no se identificaron estudiantes que correspondan al biotipo braquifacial (Tabla 1).

Tabla 1. Distribución de frecuencias para la sobremordida y el biotipo facial.

\begin{tabular}{|c|c|c|c|c|c|c|c|c|c|c|c|}
\hline & & & & \multicolumn{8}{|c|}{ MORDIDA VERTICAL } \\
\hline & & & & \multicolumn{2}{|c|}{$\begin{array}{c}\text { Mordida } \\
\text { abierta }\end{array}$} & \multicolumn{2}{|c|}{$\begin{array}{l}\text { Mordida } \\
\text { Profunda }\end{array}$} & \multicolumn{2}{|c|}{$\begin{array}{c}\text { Sobremordida } \\
\text { normal }\end{array}$} & \multirow[b]{2}{*}{$\mathbf{n}$} & \multirow[t]{2}{*}{ Total } \\
\hline & & & & $\mathbf{n}$ & $\%$ & n & $\%$ & $\mathbf{n}$ & $\%$ & & \\
\hline \multirow{12}{*}{ SEXO } & \multirow{4}{*}{ Masculino } & \multirow{4}{*}{ Biotipo } & Mesofacial & 1 & $5,0 \%$ & 8 & $40,0 \%$ & 11 & $55,0 \%$ & 20 & $100,0 \%$ \\
\hline & & & Braquifacial & 0 & $0,0 \%$ & 0 & $0,0 \%$ & 0 & $0,0 \%$ & 0 & $0,0 \%$ \\
\hline & & & Dólicofacial & 6 & $8,3 \%$ & 28 & $38,9 \%$ & 38 & $52,8 \%$ & 72 & $100,0 \%$ \\
\hline & & & Total & 7 & $7,6 \%$ & 36 & $39,1 \%$ & 49 & $53,3 \%$ & 92 & $100,0 \%$ \\
\hline & \multirow{4}{*}{ Femenino } & \multirow{4}{*}{ Biotipo } & Mesofacial & 0 & $0,0 \%$ & 2 & $20,0 \%$ & 8 & $80,0 \%$ & 10 & $100,0 \%$ \\
\hline & & & Braquifacial & 0 & $0,0 \%$ & 0 & $0,0 \%$ & 0 & $0,0 \%$ & 0 & $0,0 \%$ \\
\hline & & & Dólicofacial & 3 & $6,0 \%$ & 17 & $34,0 \%$ & 30 & $60,0 \%$ & 50 & $100,0 \%$ \\
\hline & & & Total & 3 & $5,0 \%$ & 19 & $31,7 \%$ & 38 & $63,3 \%$ & 60 & $100,0 \%$ \\
\hline & \multirow{4}{*}{ Total } & \multirow{4}{*}{ Biotipo } & Mesofacial & 1 & $3,3 \%$ & 10 & $33,3 \%$ & 19 & $63,3 \%$ & 30 & $100,0 \%$ \\
\hline & & & Braquifacial & 0 & $0,0 \%$ & 0 & $0,0 \%$ & 0 & $0,0 \%$ & 0 & $0,0 \%$ \\
\hline & & & Dólicofacial & 9 & $7,4 \%$ & 45 & $36,9 \%$ & 68 & $55,7 \%$ & 122 & $100,0 \%$ \\
\hline & & & Total & 10 & $6,6 \%$ & 55 & $36,2 \%$ & 87 & $57,2 \%$ & 152 & $100,0 \%$ \\
\hline
\end{tabular}


Tabla 2. Asociación entre el biotipo facial y la sobremordida (agrupados).

\begin{tabular}{|c|c|c|c|c|c|c|c|c|c|c|c|c|}
\hline & \multicolumn{12}{|c|}{ SOBREMORDIDA } \\
\hline & & \multicolumn{2}{|c|}{$\begin{array}{c}\text { Mordida } \\
\text { abierta }\end{array}$} & \multicolumn{2}{|c|}{$\begin{array}{l}\text { Mordida } \\
\text { profunda }\end{array}$} & \multicolumn{2}{|c|}{$\begin{array}{c}\text { Sobremordida } \\
\text { normal }\end{array}$} & \multicolumn{2}{|c|}{ Total } & \multirow[t]{2}{*}{$\mathbf{X}^{2}$} & \multirow[t]{2}{*}{$\mathbf{p}$} & \multirow[t]{2}{*}{$\mathbf{s} / \mathbf{n s}$} \\
\hline & & $\mathbf{n}$ & $\%$ & $\mathbf{n}$ & $\%$ & $\mathbf{n}$ & $\%$ & $\mathbf{n}$ & $\%$ & & & \\
\hline \multirow[t]{4}{*}{ BIOTIPO } & Mesofacial & 1 & $3,3 \%$ & 10 & $33,3 \%$ & 19 & $63,3 \%$ & 30 & 100 & & & \\
\hline & Braquifacial & 0 & $0,0 \%$ & 0 & $0,0 \%$ & 0 & $0,0 \%$ & 0 & 0 & 0,925 & 0,630 & ns \\
\hline & Dólicofacial & 9 & $7,4 \%$ & 45 & $36,9 \%$ & 68 & $55,7 \%$ & 122 & 100 & & & \\
\hline & Total & 10 & 6,6 & 55 & 36,2 & 87 & 57,2 & 152 & 100 & & & \\
\hline
\end{tabular}

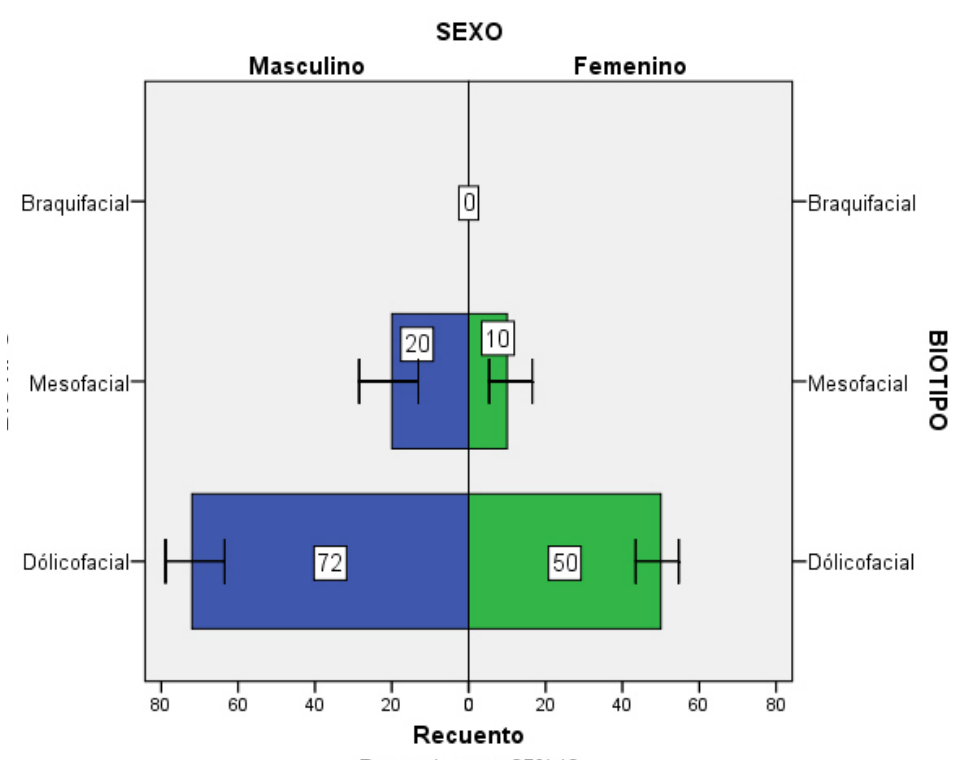

Gráfico 1. Asociación entre biotipo facial y género.

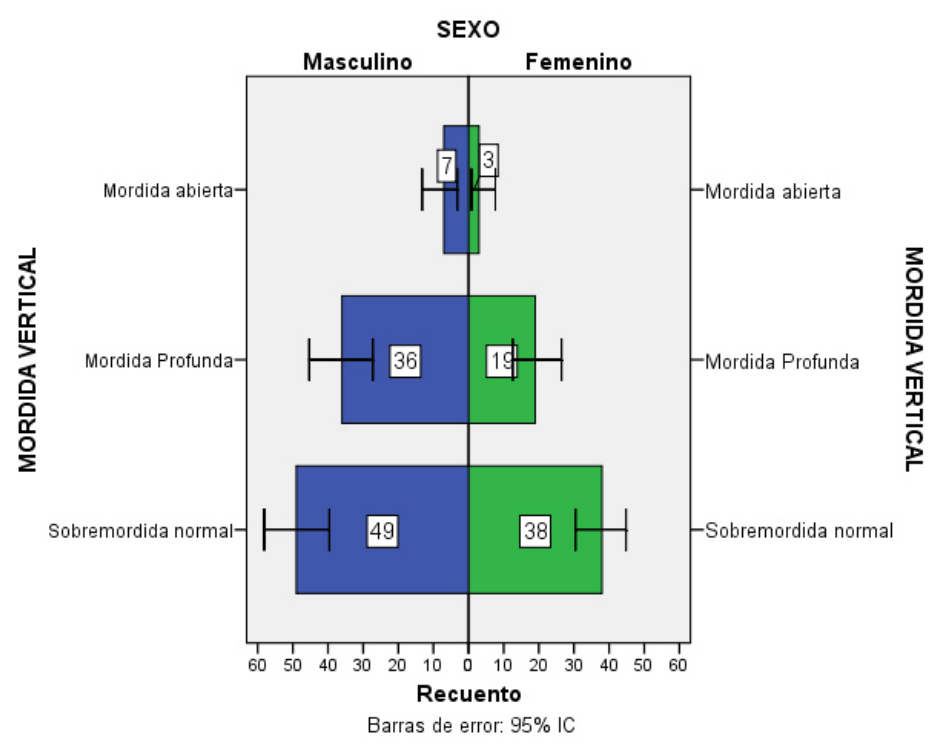

Gráfico 2. Asociación entre sobremordida y género. 
La asociación del biotipo mesofacial con la sobremordida fue la siguiente: $63,3 \%(\mathrm{n}=19)$ presentó una sobremordida normal, 33,3\% $(\mathrm{n}=10)$ mordida profunda y el $3,3 \%(n=1)$ mordida abierta. Para el grupo con biotipo dolicofacial la distribución fue: mordida normal $55,7 \%(n=68)$, mordida profunda
$36,9 \%(n=45)$ y mordida abierta 7,4\% $(n=9)$ (Tabla 2). No se encontró asociación estadísticamente significativa (prueba Xi Cuadrado; $\mathrm{p}>0,05$ ) entre las variables. La distribución de la asociación entre biotipo facial y género, sobremordida y género se muestran en los gráficos 1 y 2 respectivamente.

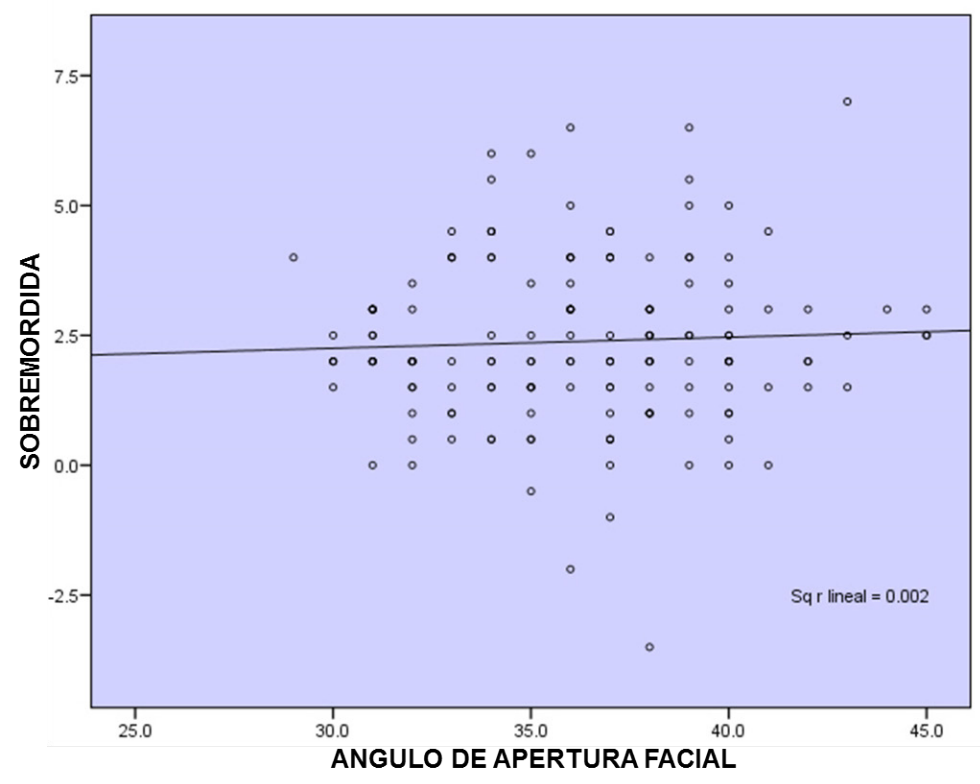

Gráfico 3. Diagrama de puntos dispersos y correlación entre los valores de sobremordida y los ángulos de apertura facial.

En el gráfico 3, se puede apreciar la correlación entre la sobremordida y los ángulos de apertura facial para la muestra estudiada.

\section{DISCUSIÓN}

A menudo el biotipo facial ha sido determinado por medio de análisis cefalométricos $(14,15)$. Arnett (16) y Farkas (17) idearon metodologías para determinar el biotipo facial por medio de un análisis del rostro en norma frontal. Farkas (17) determino el biotipo facial por medio del Indice Facial, que es una expresión de la relación entre la altura facial (NasionMentón) y el ancho bizigomático (Zygion-Zygion) en el paciente vivo. Otro método para determinar el biotipo facial es la fotometría (18). Por medio de esta técnica se puede cuantificar de manera tangible las características individuales a través de la fotografía, permitiendo ubicar de forma sencilla los puntos anatómicos que son usados como referencias para las medidas antropométricas. Esta ventaja permite aplicar la fotometría en los métodos que emplean valores de proporciones como el índice facial o medidas angulares como el ángulo de apertura facial, ya que estos valores no se verán alterados si son realizados directamente sobre el paciente o sobre una fotografía. El empleo del ángulo de apertura facial para determinar el biotipo facial ha sido usado en ortodoncia como método inicial para cuantificar las características del contorno facial de los individuos a través de fotografías $(19,20,21)$; esta caracterización permite establecer qué exámenes auxiliares serán necesarios para un diagnóstico preciso. Para el presente estudio se decidió emplear el ángulo de apertura facial como herramienta de medición del biotipo facial, debido a la imposibilidad de contar con registros radiográficos 
de la muestra estudiada; así la obtención de registros fotográficos permitió la evaluación del biotipo facial de manera sencilla y rápida. Herzberg (22) señala que las fotografías estandarizadas podrían ser el mejor método para la evaluación facial, debido a que sólo con ellas es posible evaluar en detalle medidas y proporciones. En el presente estudio la toma de las fotografías para la determinación del biotipo facial, fue realizada con los pacientes en posición natural de la cabeza $(23,24)$. Moorrees (25) señala este método como el más adecuado para el diagnóstico y tratamiento de ortodoncia ya que es reproducible en el tiempo.

Las alteraciones verticales pueden ser divididas en aquellas de origen predominantemente esquelético; cuando están relacionadas al patrón de crecimiento maxilar y mandibular; y aquellas de origen dentoalveolar, pudiendo presentarse de forma conjunta $(26,27)$. Para Hartsfield (28) la etiología de las alteraciones verticales es multifactorial, donde se asocian aspectos genéticos y ambientales; y por lo tanto, suele ser difícil determinar el grado de contribución de estos factores. Algunos estudios sugieren que para los pacientes dolicofaciales corresponderían alteraciones como la mordida abierta, y para los braquifaciales tendencia a la mordida profunda $(29,30,31,32)$. Este estudio mostró que la distribución del biotipo facial fue del $80,3 \%$ para pacientes dolicofaciales y $19,7 \%$ para los mesofaciales, no se identificaron pacientes braquifaciles. Estos datos difieren a los reportados por Carrillo y col. (33) donde el $35,45 \%$ de los pacientes fueron dolicofaciales, el $45 \%$ mesofaciales y el 19,5\% braquifaciales. Así mismo, la prevalencia de la sobremordida normal fue de $55,7 \%$, para la mordida profunda $36,9 \%$ y $7,4 \%$ para la mordida abierta. Estos autores encontraron que el $67.5 \%$ presentó mordida profunda y el $32 \%$ mordida abierta. Estos datos pueden deberse a que la muestra estudiada por Carrillo y col. (33) estuvo constituida en su totalidad por pacientes que presentaban características de alteración vertical.

La falta de asociación entre el biotipo facial y la sobremordida encontrada en el presente estudio es coherente con los resultados presentados por Otto y col. (34), quienes encontraron que el biotipo facial no influencia la extensión de la intrusión de los incisivos. Claro y col. (35) evaluaron la asociación entre la sobremordida y el patrón de crecimiento craneofacial, y no encontrando relación de dependencia entre el incremento de la sobremordida y el patrón de crecimiento braquifacial, tampoco entre la mordida abierta y el patrón de crecimiento dolicofacial. El factor determinante de la sobremordida en pacientes dolicofaciales fue la altura facial inferior, mientras que en los pacientes braquifaciales, la mayor influencia en la sobremordida fue la morfología dentoalveolar. Una sobremordida normal en las personas dolicofaciales podría ser mantenida por una compensación dentoalveolar inferior limitada (36). Algunos factores como un sistema de erupción normal y balance correcto de las fuerzas ejercidas por los tejidos blandos pueden influenciar la ocurrencia de mecanismos compensatorios $(37,38)$. La falta de asociación entre la sobremordida y el patrón facial encontrados en el estudio, sugieren que los mecanismos compensatorios podrían ser muy frecuentes.

Por tratarse de un estudio inicial, donde se evaluó el biotipo facial por medio del ángulo de apertura facial, la principal limitación fue el no poder establecer patrones esqueléticos que pudieran influenciar en la presencia de mordida abierta o mordida profunda. Sería interesante determinar en futuros estudios, el grado de confiabilidad de la evaluación clínica y fotográfica, sobre la cefalométrica del biotipo facial. En relación a los resultados de la investigación y bajo la metodología empleada se concluye que no existió una tendencia de asociación directa entre los biotipos faciales y la presencia de alteraciones verticales en la sobremordida.

\section{Correspondencia:}

Marco A. Sánchez Tito

Av. San Martín No 747, Tacna. Tacna, Perú.

Correo electrónico: marcosanchez2183@gmail.com

\section{REFERENCIAS BIBLIOGRÁFICAS}

1. Farkas LG, editor. Anthropometry of the head and face. 2nd ed. New York: Raven Press; 1994.

2. Farhad BN. Facial aesthetics concepts and clinical diagnosis. London: Wiley Blackwell. 2011.

3. Ricketts RM. The biologic significance of the divine proportion and Fibonacci series. Am J Orthod. 1982; 81(5):351-70.

4. Angle EH. Classification of malocclusion. Dent Cosmos. 1899; 41(2):248-64.

5. Ricketts RM, Planning treatment on the basis of the 
facial pattern and an estimate of its growth. The Angle Orthod. 1957; 27(1):14-37.

6. Acuña DGE, Ballesteros LM, Oropeza SG. Cephalometric description of facial patterns in skeletal open bite. Rev Odont Mex. 2013; 17(1):15-9.

7. Cozza P, Mucedero M, Baccetti T, Franchi L. Early orthodontic treatment of skeletal open bite malocclusion: a systematic review. Angle Orthod. 2005; 75(5):70713.

8. Gantz OC, Santelices BMP. Prevalencia de anomalías dentomaxilares verticales y hábitos orales disfuncionales en niños de 4 a 6 años de edad con dentición temporal completa. Rev Chil Ortod. 2013; 30(2):54-61.

9. Coben SE. The integration of facial skeletal variants. Am J Orthod. 1955; 41(6):407-34.

10. Silva Filho OG, Garib GD, Lara ST. Ortodontia interceptiva: protocolo de tratamento em duas fases. São Paulo: Artes Medicas; 2013.

11. Schwarz AM. Roentgenostatics: a pratical evaluation of the tele-X-ray-photo. New York: Leo L Bruder; 1960.

12. Jarabak JR, Fizzel JA. Technique and treatment with light wire edgewise appliances 2nd ed. Saint Louis: Mosby; 1972.

13. Bihani T. Pattern of malocclusion and treatment need in orthodontic patients in rural population: an institution based study. Adv Hum Biol. 2012; 2(3):15-22.

14. Ricketts RM. Cephalometric synthesis. Am J Dentofacial Orthod. 1960; 46(9):647-73.

15. Siriwat PP, Jarabak JR. Malocclusion and facial morphology. Is there a relationship? An epidemiologic study. Angle Orthod. 1985; 55(2):127-38.

16. Arnett GW, Bergman RT. Facial keys to orthodontic diagnosis and treatment planning: part II. Am J Orthod Dentofacial Orthop. 1993; 103(5):395-411.

17. Farkas LG, Bryson W, Klotz J. Is photogrammetry of the face reliable? Plast Reconstr Surg. 1980; 66(3):34655.

18. Martins LF, Vigorito JW. Photometric analysis applied in determining facial type. Dental Press J Orthod. 2012; 17(5):71-5.

19. Viazis AD, Atlas de ortodoncia: principios y aplicaciones clínicas. Buenos Aires: Editorial Médica Panamericana; 1995.

20. Vedovello FM, Diagnóstico dentofacial. São Paulo: Napoleão; 2011.

21. Rodríguez E, White L. Ortodoncia contemporánea: diagnóstico y tratamiento. 2da ed. México: Amolca; 2008.

22. Herzberg BL. Facial esthetic in relation to orthodontic treatment. Angle Orthod. 1952; 22(1):3-22.

23. Solow B, Tallgren A. Natural head position in standing subjects. Acta Odontol Scand. 1971; 29(5):591-607.

24. Rino Neto J, Freire-Maia BAV, Paiva JB. Método de registro de posição natural da cabeça para obtenção da radiografia cefalométrica lateral - Considerações e importância do método no diagnóstico ortodôntico- cirúrgico. Dental Press. 2003; 8(3):67-71.

25. Moorrees CF. Natural head position - a revival. Am J Orthod Dentofacial Orthop. 1994; 105(5):512-3.

26. Nielsen L. Vertical malocclusions: etiology, development, diagnosis and some aspects of treatment. Angle Orthod. $1991 ; 61(4): 247-60$.

27. Van der linder FPGM. The vertical dimension. In: McNamara Jr JA, Brudon WL. Orthodontics and dentofacial orthopedics. Needham: Ann Arbor; 2001.

28. Hartsfield J. Development of the vertical dimension: nature and nuture. Semin Orthod. 2002; 8(3):113-9.

29. Fields HW, Proffit WR, Nixon WL, Phillips C, Stanek E. Facial pattern differences in long faced children and adults. Am J Orthod. 1984; 85(3):217-23.

30. Keeling SD, Riolo ML, Martin RE, Ten Have TR. A multivariate approach to analyzing the relation between occlusion and craniofacial morphology. Am J Orthod Dentofacial Orthop. 1989; 95(4):297-305.

31. Boom HPW, van Spronsen PH, van Ginkel FC, van Schijndel RA, Castelijns JA, Tuinzing DB. A comparison of human jaw muscle cross-sectional area and volume in long- and short-face subjects, using MRI. Arch Oral Biol. 2008; 53(3):273-81.

32. Andrade J. Avaliação do Biotipo Facial em Pacientes com espiração Oral ou Mista [Tesis Mestrado].Portugal. Universidade Católica Portuguesa; 2011.

33. Carrillo PG, Córdoba GN, Correa MV, Vera RA, Bastidas RCL, Perdomo OAF. Prevalencia de las maloclusiones verticales en pacientes tratados en la Universidad Cooperativa de Colombia. Rev Nal Odo UCC. 2008; 4(7):24-31.

34. Otto RL, Anholm JM, Engel GA. A comparative analysis of intrusion of incisor teeth achieved in adults and children according to facial type. Am J Orthod. 1980; 77(4):437-46.

35. Claro CAA, Abrão J, Reis SAB. Association between overbite and craniofacial growth pattern. Braz Oral Res. 2010; 24(4):425-32.

36. Kuitert R, Beckmann S, Loenen M, Tuinzing B, Zentner A. Dentoalveolar compensation in subjects with vertical skeletal dysplasia. Am J Orthod Dentofacial Orthop. 2006; 129(5):649-57.

37. Solow B. The dentoalveolar compensatory mechanism: background and clinical implications. $\mathrm{Br} \mathrm{J}$ Orthod. 1980; 7(3):145-61.

38. Proffit WR. Equilibrium theory revisited: factors influencing position of the teeth. Angle Orthod. 1978; 48(3)175-86.

Recibido: 18/12/2014

Aceptado: 20/02/2015 